\title{
Absolute stability of large-scale time-delay Lurie indirect control systems with unbounded coefficients
}

\section{Xiao Yu and Fucheng Liao*}

\section{"Correspondence:}

fcliao@ustb.edu.cn

School of Mathematics and Physics,

University of Science and

Technology Beijing, Beijing, 100083,

China

\begin{abstract}
We study the absolute stability of large-scale time-delay Lurie indirect control systems with unbounded coefficients. Based on Lyapunov-Krasovskii functional approach, we derive some novel absolute stability conditions for this class of Lurie systems with a single nonlinearity. These conditions are particularly suitable for large-scale time-delay Lurie systems with unbounded coefficients. At the same time, they are also effective for such systems with bounded or constant coefficients. Furthermore, we extend the results obtained to multiple nonlinearities. The effectiveness of the proposed methods is illustrated via two numerical examples.
\end{abstract}

Keywords: large-scale time-delay system; lurie indirect control system; absolute stability; Lyapunov second method

\section{Introduction}

In the early 1940s, the absolute stability concept was defined by Lurie and Postnikov [1]. Since then, the absolute stability analysis for Lurie systems has received considerable attention from the academic community, and there have been a great number of publications on this topic [2-6]. Meanwhile, the time-delay phenomenon frequently appears in practical engineering systems. Its presence can degrade performance of the control system or even lead to instability. Therefore, the research of absolute stability of time-delay Lurie systems is of important significance. In [7], by splitting the whole delay interval into even or uneven subintervals, a new Lyapunov-Krasovskii functional was constructed. Based on Lyapunov second method, some new absolute stability conditions were proposed. In [8], by employing integral-equality technique, the stability criteria for Lurie control systems with multiple delays were obtained. In [9], the absolute stability of Lurie nonlinear systems with time-varying delay was investigated based on augmented Lyapunov-Krasovskii functional and free-weighting matrix approach. At the same time, the absolute stability theory of time-delay Lurie systems was also applied in the study of synchronization problems; some relevant works were presented in [10-14].

It should be pointed out that some practical systems, such as communication systems, electric power systems, and biological systems, can be represented in the form of largescale systems [15]. Over the past few years, much effort has been devoted to investigating the problem of absolute stability of large-scale Lurie control systems, and many impor-

(c) The Author(s) 2017. This article is distributed under the terms of the Creative Commons Attribution 4.0 International License (http://creativecommons.org/licenses/by/4.0/), which permits unrestricted use, distribution, and reproduction in any medium, provided you give appropriate credit to the original author(s) and the source, provide a link to the Creative Commons license, and indicate if changes were made. 
tant results have been reported in the open literature. In [16], some sufficient conditions in terms of BMIs were presented to guarantee the absolute stability of interconnected Lurie direct control system. By using the decomposing method of large-scale system and M-matrix property, the authors in [17] considered a class of Lurie indirect control largescale systems and derived simple stability conditions. Subsequently, the authors in [18] extended the criteria proposed in [17] to the case of large-scale Lurie indirect control systems with multiple operators and unbounded coefficients. By employing a similar method, the absolute stability problem of large-scale Lurie direct control systems with time-varying coefficients was well addressed in $[19,20]$. However, to our knowledge, there are few results on the absolute stability of large-scale Lurie systems subject to time-delay. This has motivated our study.

In this paper, we deal with the absolute stability problem of a class of large-scale timedelay Lurie indirect control systems with unbounded coefficients. A Lyapunov-Krasovskii functional-based approach is presented to obtain some new sufficient conditions such that the absolute stability of the system under consideration can be ensured. The main contributions of this paper are as follows: (i) The elements of the system coefficient matrices can be unbounded functions, and the time-delay can be very large under admissible conditions. (ii) The stability criteria proposed are applicable not only to large-scale time-delay Lurie systems with unbounded coefficients but also to this class of systems with bounded or constant coefficients.

Notation: Throughout the paper, $P>0(P<0)$ means that a matrix $P$ is symmetric positive (negative) definite; $\lambda(A)$ denotes any eigenvalue of square matrix $A$; $\|x\|$ stands for the Euclidean norm of a vector $x=\left[\begin{array}{llll}x_{1} x_{2} & \cdots & x_{n}\end{array}\right]^{T}$, that is, $\|x\|=\sqrt{\sum_{i=1}^{n} x_{i}^{2}}$; $\|A\|$ is the matrix norm induced by the vector Euclidean norm, that is, $\|A\|=\max _{\|x\|=1}\|A x\|$, and it is easy to verify that $\|A\|=\sqrt{\lambda_{\max }\left(A^{T} A\right)} ; \varlimsup_{\lim _{t \rightarrow \infty}}$ denotes the upper limit. For simplicity of presentation, let $\phi(s)=\left[x_{1}^{T}(t+s) x_{2}^{T}(t+s) \cdots x_{m}^{T}(t+s) \sigma(t)\right]^{T}, s \in[-\tau, 0], t \geq 0$; $|\|\phi\||=\sqrt{\int_{-\tau}^{0}\|\phi(s)\|^{2} d s}$.

To obtain our main results, the following lemmas are needed.

Lemma 1 (The Schur complement lemma [21]) Let $M, N, P$ be constant matrices of appropriate dimensions, where $M$ and $N$ are symmetric. Then

$$
\left[\begin{array}{ll}
M & P \\
P^{T} & N
\end{array}\right]<0
$$

if and only if $N<0$ and $M-P N^{-1} P^{T}<0$.

Consider the following functional differential equations with finite delay:

$$
\dot{x}(t)=f\left(t, x_{t}\right)
$$

where $x(t) \in R^{n}, x_{t}$ is a function defined in the interval $[-h, 0]$ as $x_{t}(\theta)=x(t+\theta),-h \leq \theta \leq 0$, $h$ is the maximum delay, and $f$ is a functional. Let $C$ be the set of all continuous functions defined in the interval $[-h, 0]$. Then the initial condition of (1) can be expressed as $x_{t_{0}}=\phi$, for $\phi \in C$. With this notation, the domain of definition of $f$ is $R \times C$. A more detailed description can be found in [22]. 
Denote by $K$ the set of strictly increasing continuous functions $W: R_{+} \rightarrow R_{+}$with $W(0)=0$. Also, $|\|\phi\||=\sqrt{\int_{-\tau}^{0}\|\phi(s)\|^{2} d s}$, where $\|\cdot\|$ refers to the usual Euclidean norm. For system (1), we have the following lemma.

Lemma 2 Suppose that a functional $V$ and functions $W, W_{1}, W_{2}, W_{3} \in K$ are such that

(i) $W(\|\phi(0)\|) \leq V(t, \phi) \leq W_{1}(\|\phi(0)\|)+W_{2}(|\|\phi\||)$,

(ii) $\dot{V}(t, \phi) \leq-W_{3}(\|\phi(0)\|)$.

Then the zero solution of (1) is uniformly asymptotically stable. In addition, if $\lim _{s \rightarrow \infty} W(s)=\infty$, then it is globally uniformly asymptotically stable.

Proof Burton in $[22,23]$ has proved the classical uniform asymptotic stability result. In fact, if $\lim _{s \rightarrow \infty} W(s)=\infty$, then, in the proof of Theorem 1 of [23], for arbitrary large $\delta>0$, there exists $\varepsilon>0$ such that $W_{1}(\delta)+W_{2}\left(\left[n h \delta^{2}\right]^{\frac{1}{2}}\right)<W(\varepsilon)$, and therefore the global uniform asymptotic stability can be concluded.

We first analyze large-scale time-delay Lurie indirect control systems with a single nonlinearity, and then we extend the results derived to multiple nonlinearities. For the multiple nonlinearities case, $\sigma(t)$ in $\phi(s)$ is regarded as a vector.

\section{Absolute stability of large-scale Lurie systems with a single nonlinearity}

Consider the following large-scale time-delay Lurie indirect control system with unbounded coefficients and a single nonlinearity:

$$
\left\{\begin{array}{l}
\dot{x}_{i}(t)=\sum_{j=1}^{m} A_{i j}(t) x_{j}(t)+\sum_{j=1}^{m} B_{i j}(t) x_{j}\left(t-\tau_{j}(t)\right)+b_{i}(t) f(\sigma(t)), \\
\quad i=1,2, \ldots, m \\
\dot{\sigma}(t)=\sum_{i=1}^{m} c_{i}^{T}(t) x_{i}(t)-\rho(t) f(\sigma(t)),
\end{array}\right.
$$

where $x_{i}(t) \in R^{n_{i}}(i=1,2, \ldots, m)$ and $\sigma(t) \in R$ are the state vectors, the vector functions $b_{i}(t), c_{i}(t) \in R^{n_{i}}(i=1,2, \ldots, m)$ are continuous in $[0, \infty), \sum_{i=1}^{m} n_{i}=n$; the matrix functions $A_{i j}(t), B_{i j}(t) \in R^{n_{i} \times n_{j}}(i, j=1,2, \ldots, m)$ are continuous in $[0, \infty) ; \tau_{j}(t)(j=1,2, \ldots, m)$ refers to the time-delay; $\rho(t)$ is a continuous function in $[0, \infty)$ and satisfies $\rho(t) \geq \rho>0$ with constant $\rho$. The continuous nonlinearity $f(\cdot)$ satisfies the following sector condition:

$$
F_{\left[k_{1}, k_{2}\right]}=\left\{f(\cdot) \mid f(0)=0 ; k_{1} \sigma^{2}(t) \leq \sigma(t) f(\sigma(t)) \leq k_{2} \sigma^{2}(t), \sigma(t) \in R-\{0\}\right\}
$$

where $k_{1}, k_{2}$ are constants such that $k_{2}>k_{1}>0$.

Definition 1 ([24]) System (2) is said to be absolutely stable if its zero solution is globally asymptotically stable for any nonlinearity $f(\cdot) \in F_{\left[k_{1}, k_{2}\right]}$.

We make the following assumptions for system (2).

A1 The time-delay $\tau_{i}(t)(i=1,2, \ldots, m)$ are continuous and piecewise differentiable functions with

$$
0 \leq \tau_{i}(t) \leq \tau_{i}, \quad \dot{\tau}_{i}(t) \leq \alpha_{i}<1
$$


where $\tau_{i}, \alpha_{i}(i=1,2, \ldots, m)$ are constants. At the nondifferentiability points of $\tau_{i}(t)$, $\dot{\tau}_{i}(t)$ represents $\max \left[\dot{\tau}_{i}(t-0), \dot{\tau}_{i}(t+0)\right]$.

A2 For any $t \in[0, \infty)$, there exist matrices $P_{i}>0, G_{i}>0(i=1,2, \ldots, m)$ such that

$$
\lambda\left(P_{i} A_{i i}(t)+A_{i i}^{T}(t) P_{i}+G_{i}\right) \leq-\delta_{i}(t) \leq-\xi_{i}<0,
$$

where $\delta_{i}(t)>0, \xi_{i}>0(i=1,2, \ldots, m)$ are functions and constants, respectively.

A3 For any $t \in[0, \infty)$,

$$
\frac{\left\|P_{i} A_{i j}(t)+A_{j i}^{T}(t) P_{j}\right\|}{\sqrt{\delta_{i}(t) \delta_{j}(t)}} \leq \eta_{i j}, \quad \frac{\left\|P_{i} B_{i j}(t)\right\|}{\sqrt{\delta_{i}(t)\left(1-\alpha_{j}\right) \lambda_{\min }\left(G_{j}\right)}} \leq \gamma_{i j},
$$

where $\eta_{i j}(i, j=1,2, \ldots, m ; i \neq j), \gamma_{i j}(i, j=1,2, \ldots, m)$ are constants, and $\eta_{i j}=\eta_{j i}$. A4 For any $t \in[0, \infty)$,

$$
\frac{\left\|P_{i} b_{i}(t)+\frac{1}{2} c_{i}(t)\right\|}{\sqrt{\delta_{i}(t) \rho(t)}} \leq \mu_{i}
$$

where $\mu_{i}(i=1,2, \ldots, m)$ are constants.

To simplify the statements, we define the following auxiliary matrices:

$$
D=\left[\begin{array}{cccc}
-1 & \eta_{12} & \cdots & \eta_{1 m} \\
\eta_{21} & -1 & \cdots & \eta_{2 m} \\
\vdots & \vdots & & \vdots \\
\eta_{m 1} & \eta_{m 2} & \cdots & -1
\end{array}\right], \quad R=\left[\begin{array}{cccc}
\gamma_{11} & \gamma_{12} & \cdots & \gamma_{1 m} \\
\gamma_{21} & \gamma_{22} & \cdots & \gamma_{2 m} \\
\vdots & \vdots & & \vdots \\
\gamma_{m 1} & \gamma_{m 2} & \cdots & \gamma_{m m}
\end{array}\right], \quad U=\left[\begin{array}{c}
\mu_{1} \\
\mu_{2} \\
\vdots \\
\mu_{m}
\end{array}\right]
$$

Notice that by A3 the matrix $D$ is symmetric.

Theorem 1 Under A1-A4, system (2) is absolutely stable if $D+R R^{T}+U U^{T}<0$.

Proof By utilizing $P_{i}, G_{i}(i=1,2, \ldots, m)$ appearing in the assumptions, we choose the following Lyapunov-Krasovskii functional:

$$
V(t, \phi)=\sum_{i=1}^{m}\left(x_{i}^{T}(t) P_{i} x_{i}(t)+\int_{t-\tau_{i}(t)}^{t} x_{i}^{T}(s) G_{i} x_{i}(s) d s\right)+\int_{0}^{\sigma(t)} f(s) d s .
$$

We can verify that if $f \in F_{\left[k_{1}, k_{2}\right]}$, then $\frac{1}{2} k_{1} \sigma^{2}(t) \leq \int_{0}^{\sigma(t)} f(s) d s \leq \frac{1}{2} k_{2} \sigma^{2}(t)$. Letting $\tau=$ $\max \left\{\tau_{i}, i=1,2, \ldots, m\right\}, V(t, \phi)$ satisfies

$$
\begin{aligned}
& \sum_{i=1}^{m} \lambda_{\min }\left(P_{i}\right)\left\|x_{i}(t)\right\|^{2}+\frac{1}{2} k_{1} \sigma^{2}(t) \\
& \quad \leq V(t, \phi) \leq \sum_{i=1}^{m}\left(\lambda_{\max }\left(P_{i}\right)\left\|x_{i}(t)\right\|^{2}+\lambda_{\max }\left(G_{i}\right) \int_{t-\tau}^{t}\left\|x_{i}(s)\right\|^{2} d s\right)+\frac{1}{2} k_{2} \sigma^{2}(t)
\end{aligned}
$$


Further, we have

$$
\begin{aligned}
& \min \left\{\lambda_{\min }\left(P_{1}\right), \ldots, \lambda_{\min }\left(P_{m}\right), \frac{1}{2} k_{1}\right\}\|\phi(0)\|^{2} \\
& \leq V(t, \phi) \\
& \leq \max \left\{\lambda_{\max }\left(P_{1}\right), \ldots, \lambda_{\max }\left(P_{m}\right), \frac{k_{2}}{2}\right\}\|\phi(0)\|^{2} \\
& \quad+\max \left\{\lambda_{\max }\left(G_{1}\right), \ldots, \lambda_{\max }\left(G_{m}\right)\right\} \int_{-\tau}^{0}\|\phi(s)\|^{2} d s
\end{aligned}
$$

Namely, let

$$
\begin{aligned}
& W_{1}(s)=\min \left\{\lambda_{\min }\left(P_{1}\right), \ldots, \lambda_{\min }\left(P_{m}\right), \frac{1}{2} k_{1}\right\} s^{2}, \\
& W_{2}(s)=\max \left\{\lambda_{\max }\left(P_{1}\right), \ldots, \lambda_{\max }\left(P_{m}\right), \frac{1}{2} k_{2}\right\} s^{2}, \\
& W_{3}(s)=\max \left\{\lambda_{\max }\left(G_{1}\right), \ldots, \lambda_{\max }\left(G_{m}\right)\right\} s^{2} .
\end{aligned}
$$

Then we obtain, for $t \geq 0$, the following inequalities:

$$
W_{1}(\|\phi(0)\|) \leq V(t, \phi) \leq W_{2}(\|\phi(0)\|)+W_{3}(|\|\phi\||) .
$$

Thus, condition (i) of Lemma 2 is satisfied. Moreover, $\lim _{s \rightarrow \infty} W_{1}(s)=\infty$.

The derivative of $V(t, \phi)$ along the trajectory of system (2) is

$$
\begin{aligned}
\left.\dot{V}(t, \phi)\right|_{(2)} & \\
= & \sum_{i=1}^{m}\left(2 x_{i}^{T}(t) P_{i} \dot{x}_{i}(t)+x_{i}^{T}(t) G_{i} x_{i}(t)\right) \\
& -\sum_{i=1}^{m}\left(1-\dot{\tau}_{i}(t)\right) x_{i}^{T}\left(t-\tau_{i}(t)\right) G_{i} x_{i}\left(t-\tau_{i}(t)\right)+\dot{\sigma}(t) f(\sigma(t)) \\
= & \sum_{i=1}^{m} x_{i}^{T}(t)\left(P_{i} A_{i i}(t)+A_{i i}^{T}(t) P_{i}+G_{i}\right) x_{i}(t)+2 \sum_{i=1}^{m} \sum_{j=1}^{m} x_{i}^{T}(t) P_{i} A_{i j}(t) x_{j}(t) \\
& +2 \sum_{i=1}^{m} \sum_{j=1}^{m} x_{i}^{T}(t) P_{i} B_{i j}(t) x_{j}\left(t-\tau_{j}(t)\right)+2 \sum_{i=1}^{m} x_{i}^{T}(t) P_{i} b_{i}(t) f(\sigma(t)) \\
& -\sum_{i=1}^{m}\left(1-\dot{\tau}_{i}(t)\right) x_{i}^{T}\left(t-\tau_{i}(t)\right) G_{i} x_{i}\left(t-\tau_{i}(t)\right)+\sum_{i=1}^{m} x_{i}^{T}(t) c_{i}(t) f(\sigma(t)) \\
& -\rho(t) f^{2}(\sigma(t)) \\
& m \\
= & \sum_{i=1}^{m} x_{i}^{T}(t)\left(P_{i} A_{i i}(t)+A_{i i}^{T}(t) P_{i}+G_{i}\right) x_{i}(t)+2 \sum_{i=1}^{m} \sum_{j=1}^{m} x_{i}^{T}(t) P_{i} A_{i j}(t) x_{j}(t) \\
j \neq i &
\end{aligned}
$$




$$
\begin{aligned}
& +2 \sum_{i=1}^{m} \sum_{j=1}^{m} x_{i}^{T}(t) P_{i} B_{i j}(t) x_{j}\left(t-\tau_{j}(t)\right)+2 \sum_{i=1}^{m} x_{i}^{T}(t)\left(P_{i} b_{i}(t)+\frac{1}{2} c_{i}(t)\right) f(\sigma(t)) \\
& -\sum_{i=1}^{m}\left(1-\dot{\tau}_{i}(t)\right) x_{i}^{T}\left(t-\tau_{i}(t)\right) G_{i} x_{i}\left(t-\tau_{i}(t)\right)-\rho(t) f^{2}(\sigma(t)) \\
= & \sum_{i=1}^{m} x_{i}^{T}(t)\left(P_{i} A_{i i}(t)+A_{i i}^{T}(t) P_{i}+G_{i}\right) x_{i}(t)+\sum_{i=1}^{m} \sum_{\substack{j=1 \\
j \neq i}}^{m} x_{i}^{T}(t)\left(P_{i} A_{i j}(t)+A_{j i}^{T}(t) P_{j}\right) x_{j}(t) \\
& +2 \sum_{i=1}^{m} \sum_{j=1}^{m} x_{i}^{T}(t) P_{i} B_{i j}(t) x_{j}\left(t-\tau_{j}(t)\right)+2 \sum_{i=1}^{m} x_{i}^{T}(t)\left(P_{i} b_{i}(t)+\frac{1}{2} c_{i}(t)\right) f(\sigma(t)) \\
& -\sum_{i=1}^{m}\left(1-\dot{\tau}_{i}(t)\right) x_{i}^{T}\left(t-\tau_{i}(t)\right) G_{i} x_{i}\left(t-\tau_{i}(t)\right)-\rho(t) f^{2}(\sigma(t)) .
\end{aligned}
$$

From $\mathrm{A} 1$ and $\mathrm{A} 2$, using properties of the matrix norm, we get

$$
\begin{aligned}
\left.\dot{V}(t, \phi)\right|_{(2)} & \\
\leq & \sum_{i=1}^{m} x_{i}^{T}(t)\left(P_{i} A_{i i}(t)+A_{i i}^{T}(t) P_{i}+G_{i}\right) x_{i}(t)+\sum_{i=1}^{m} \sum_{\substack{j=1 \\
j \neq i}}^{m} x_{i}^{T}(t)\left(P_{i} A_{i j}(t)+A_{j i}^{T}(t) P_{j}\right) x_{j}(t) \\
& +2 \sum_{i=1}^{m} \sum_{j=1}^{m} x_{i}^{T}(t) P_{i} B_{i j}(t) x_{j}\left(t-\tau_{j}(t)\right)+2 \sum_{i=1}^{m} x_{i}^{T}(t)\left(P_{i} b_{i}(t)+\frac{1}{2} c_{i}(t)\right) f(\sigma(t)) \\
& -\sum_{i=1}^{m}\left(1-\alpha_{i}\right) x_{i}^{T}\left(t-\tau_{i}(t)\right) G_{i} x_{i}\left(t-\tau_{i}(t)\right)-\rho(t) f^{2}(\sigma(t)) \\
\leq & -\sum_{i=1}^{m} \delta_{i}(t)\left\|x_{i}(t)\right\|^{2}+\sum_{i=1}^{m} \sum_{j=1}^{m}\left\|P_{i} A_{i j}(t)+A_{j i}^{T}(t) P_{j}\right\|\left\|x_{i}(t)\right\|\left\|x_{j}(t)\right\| \\
& +2 \sum_{i=1}^{m} \sum_{j=1}^{m}\left\|P_{i} B_{i j}(t)\right\|\left\|x_{i}(t)\right\|\left\|x_{j}\left(t-\tau_{j}(t)\right)\right\| \\
& +2 \sum_{i=1}^{m}\left\|P_{i} b_{i}(t)+\frac{c_{i}(t)\|\| x_{i}(t) \||f(\sigma(t))|}{2}\right\| \lambda^{m}\left(1-\alpha_{i}\right) \lambda_{\min }\left(G_{i}\right)\left\|x_{i}\left(t-\tau_{i}(t)\right)\right\|^{2}-\rho(t) f^{2}(\sigma(t)) .
\end{aligned}
$$

To fully utilize A3, A4, and the unbounded terms in system coefficients, we take $\sqrt{\delta_{i}(t)}\left\|x_{i}(t)\right\|, \sqrt{\left(1-\alpha_{i}\right) \lambda_{\min }\left(G_{i}\right)}\left\|x\left(t-\tau_{i}(t)\right)\right\|(i=1,2, \ldots, m)$, and $\sqrt{\rho(t)}|f(\sigma(t))|$ as the variables of the following quadratic form. Then the inequality becomes

$$
\begin{aligned}
& \left.\dot{V}(t, \phi)\right|_{(2)} \\
& \quad \leq-\sum_{i=1}^{m} \delta_{i}(t)\left\|x_{i}(t)\right\|^{2}+\sum_{\substack { i=1 \\
\begin{subarray}{c}{j=1 \\
j \neq i{ i = 1 \\
\begin{subarray} { c } { j = 1 \\
j \neq i } }\end{subarray}}^{m} \frac{\left\|P_{i} A_{i j}(t)+A_{j i}^{T}(t) P_{j}\right\|}{\sqrt{\delta_{i}(t) \delta_{j}(t)}} \sqrt{\delta_{i}(t)}\left\|x_{i}(t)\right\| \cdot \sqrt{\delta_{j}(t)}\left\|x_{j}(t)\right\|
\end{aligned}
$$




$$
\begin{aligned}
& +2 \sum_{i=1}^{m} \sum_{j=1}^{m} \frac{\left\|P_{i} B_{i j}(t)\right\|}{\sqrt{\delta_{i}(t)\left(1-\alpha_{j}\right) \lambda_{\min }\left(G_{j}\right)}} \sqrt{\delta_{i}(t)}\left\|x_{i}(t)\right\| \cdot \sqrt{\left(1-\alpha_{j}\right) \lambda_{\min }\left(G_{j}\right)} x_{j}\left(t-\tau_{j}(t)\right) \\
& +2 \sum_{i=1}^{m} \frac{\left\|P_{i} b_{i}(t)+\frac{1}{2} c_{i}(t)\right\|}{\sqrt{\delta_{i}(t) \rho(t)}} \sqrt{\delta_{i}(t)}\left\|x_{i}(t)\right\| \cdot \sqrt{\rho(t)}|f(\sigma(t))| \\
& -\sum_{i=1}^{m}\left(1-\alpha_{i}\right) \lambda_{\min }\left(G_{i}\right)\left\|x_{i}\left(t-\tau_{i}(t)\right)\right\|^{2}-\rho(t) f^{2}(\sigma(t)) \\
& \leq-\sum_{i=1}^{m} \delta_{i}(t)\left\|x_{i}(t)\right\|^{2}+\sum_{i=1}^{m} \sum_{j=1}^{m} \eta_{i j} \sqrt{\delta_{i}(t)}\left\|x_{i}(t)\right\| \cdot \sqrt{\delta_{j}(t)}\left\|x_{j}(t)\right\| \\
& +\sum_{i=1}^{m} \sum_{j=1}^{m} 2 \gamma_{i j} \sqrt{\delta_{i}(t)}\left\|x_{i}(t)\right\| \cdot \sqrt{\left(1-\alpha_{j}\right) \lambda_{\min }\left(G_{j}\right)}\left\|x_{j}\left(t-\tau_{j}(t)\right)\right\| \\
& +\sum_{i=1}^{m} 2 \mu_{i} \sqrt{\delta_{i}(t)}\left\|x_{i}(t)\right\| \cdot \sqrt{\rho(t)}|f(\sigma(t))|-\sum_{i=1}^{m}\left(1-\alpha_{i}\right) \lambda_{\min }\left(G_{i}\right)\left\|x_{i}\left(t-\tau_{i}(t)\right)\right\|^{2} \\
& -\rho(t) f^{2}(\sigma(t)) \\
& =Y^{T} \hat{D} Y
\end{aligned}
$$

where

$$
\begin{aligned}
& Y=\left[\begin{array}{c}
\sqrt{\delta_{1}(t)}\left\|x_{1}(t)\right\| \\
\vdots \\
\sqrt{\delta_{m}(t)}\left\|x_{m}(t)\right\| \\
\sqrt{\left(1-\alpha_{1}\right) \lambda_{\min }\left(G_{1}\right)}\left\|x_{1}\left(t-\tau_{1}(t)\right)\right\| \\
\vdots \\
\sqrt{\left(1-\alpha_{m}\right) \lambda_{\min }\left(G_{m}\right)}\left\|x_{m}\left(t-\tau_{m}(t)\right)\right\| \\
\sqrt{\rho(t)}|f(\sigma(t))|
\end{array}\right], \\
& \hat{D}=\left[\begin{array}{ccccccccc}
-1 & \eta_{12} & \cdots & \eta_{1 m} & \gamma_{11} & \gamma_{12} & \cdots & \gamma_{1 m} & \mu_{1} \\
\eta_{21} & -1 & \cdots & \eta_{2 m} & \gamma_{21} & \gamma_{22} & \cdots & \gamma_{2 m} & \mu_{2} \\
\vdots & \vdots & & \vdots & \vdots & \vdots & & \vdots & \vdots \\
\eta_{m 1} & \eta_{m 2} & \cdots & -1 & \gamma_{m 1} & \gamma_{m 2} & \cdots & \gamma_{m m} & \mu_{m} \\
\gamma_{11} & \gamma_{21} & \cdots & \gamma_{m 1} & -1 & 0 & \cdots & 0 & 0 \\
\gamma_{12} & \gamma_{22} & \cdots & \gamma_{m 2} & 0 & -1 & \cdots & 0 & 0 \\
\vdots & \vdots & & \vdots & \vdots & \vdots & & \vdots & \vdots \\
\gamma_{1 m} & \gamma_{2 m} & \cdots & \gamma_{m m} & 0 & 0 & \cdots & -1 & 0 \\
\mu_{1} & \mu_{2} & \cdots & \mu_{m} & 0 & 0 & \cdots & 0 & -1
\end{array}\right] .
\end{aligned}
$$

Using the preceding notations, we have

$$
\hat{D}=\left[\begin{array}{ccc}
D & R & U \\
R^{T} & -I & 0 \\
U^{T} & 0 & -1
\end{array}\right] .
$$


By Lemma $1, D+R R^{T}+U U^{T}<0$ implies $\hat{D}<0$. Let $-\beta(\beta>0)$ be the maximum eigenvalue of $\hat{D}$. Then, $\left.\dot{V}(t, \phi)\right|_{(2)}$ satisfies

$$
\begin{aligned}
& \left.\dot{V}(t, \phi)\right|_{(2)} \\
& \quad \leq-\beta\left(\sum_{i=1}^{m}\left(\delta_{i}(t)\left\|x_{i}(t)\right\|^{2}+\left(1-\alpha_{i}\right) \lambda_{\min }\left(G_{i}\right)\left\|x_{i}\left(t-\tau_{i}(t)\right)\right\|^{2}\right)+\rho(t)|f(\sigma(t))|^{2}\right) \\
& \quad \leq-\beta\left(\sum_{i=1}^{m} \xi_{i}\left\|x_{i}(t)\right\|^{2}+\rho|f(\sigma(t))|^{2}\right) .
\end{aligned}
$$

Since $\sigma(t) f(\sigma(t)) \geq k_{1} \sigma^{2}(t)$, we obtain $|f(\sigma(t))| \geq k_{1}|\sigma(t)|$. Hence,

$$
\begin{aligned}
& \left.\dot{V}(t, \phi)\right|_{(2)} \\
& \quad \leq-\beta\left(\sum_{i=1}^{m} \xi_{i}\left\|x_{i}(t)\right\|^{2}+\rho k_{1}^{2} \sigma^{2}(t)\right) \\
& \quad \leq-\beta \min \left\{\xi_{1}, \ldots, \xi_{m}, \rho k_{1}^{2}\right\}\left(\sum_{i=1}^{m}\left\|x_{i}(t)\right\|^{2}+\sigma^{2}(t)\right) .
\end{aligned}
$$

Letting $W_{4}(s)=-\beta \min \left\{\xi_{1}, \ldots, \xi_{m}, \rho k_{1}^{2}\right\} s^{2}$, we have

$$
\left.\dot{V}(t, \phi)\right|_{(2)} \leq W_{4}(\|\phi(0)\|)
$$

This means that condition (ii) of Lemma 2 is satisfied. Thus, by Lemma 2 and Definition 1 , system (2) is absolutely stable. This completes the proof.

A5 For any $t \in[0, \infty)$, there exist matrices $P_{i}>0, G_{i}>0(i=1,2, \ldots, m)$ such that

$$
\lambda\left(P_{i} A_{i i}(t)+A_{i i}^{T}(t) P_{i}+G_{i}\right) \leq-\delta_{i}(t)<0,
$$

where $\delta_{i}(t)>0(i=1,2, \ldots, m)$. Let $\delta(t)=\min \left\{\delta_{1}(t), \delta_{2}(t), \ldots, \delta_{m}(t)\right\}$ and assume that $\lim _{t \rightarrow \infty} \delta(t)=\infty$.

Corollary 1 Under A1, A3, A4, and A5, system (2) is absolutely stable if $D+R R^{T}+$ $U U^{T}<0$.

Indeed, by exploiting the limit property, we derive from $\lim _{t \rightarrow \infty} \delta(t)=\infty$ that, for any $\xi_{i}>0(i=1,2, \ldots, m)$ (here let $\left.\xi_{i}=1\right)$, there exists $T \geq 0$ such that, for $t>T$,

$$
-\delta(t) \leq-\xi_{i}
$$

This implies that

$$
\lambda\left(P_{i} A_{i i}(t)+A_{i i}^{T}(t) P_{i}+G_{i}\right) \leq-\delta_{i}(t) \leq-\delta(t)<-\xi_{i} .
$$

The result then follows immediately from Theorem 1. 
In fact, we only need to ensure that the conditions mentioned are satisfied when time $t$ is sufficiently large, because asymptotic stability refers to the behavior of the dynamic systems as time tends to infinity. In other words, A2-A5 can be written as follows: There exists $T \geq 0$ such that the corresponding conditions are satisfied for $t>T$. In particular, $\mathrm{A} 3$ and A4 can be rewritten as follows.

A6

$$
\varlimsup_{t \rightarrow \infty} \frac{\left\|P_{i} A_{i j}(t)+A_{j i}^{T}(t) P_{j}\right\|}{\sqrt{\delta_{i}(t) \delta_{j}(t)}}=\eta_{i j}, \quad \varlimsup_{t \rightarrow \infty} \frac{\left\|P_{i} B_{i j}(t)\right\|}{\sqrt{\delta_{i}(t)\left(1-\alpha_{j}\right) \lambda_{\min }\left(G_{j}\right)}}=\gamma_{i j},
$$

where $\eta_{i j}(i, j=1,2, \ldots, m ; i \neq j)$ and $\gamma_{i j}(i, j=1,2, \ldots, m)$ are constants, and $\eta_{i j}=\eta_{j i}$. A7

$$
\varlimsup_{t \rightarrow \infty} \frac{\left\|P_{i} b_{i}(t)+\frac{1}{2} c_{i}(t)\right\|}{\sqrt{\delta_{i}(t) \rho(t)}}=\mu_{i}
$$

where $\mu_{i}(i=1,2, \ldots, m)$ are constants.

Corollary 2 Under A1, A2, A6, and A7, system (2) is absolutely stable if $D+R R^{T}+$ $U U^{T}<0$.

Corollary 3 Under A1, A2, A6, and A7, system (2) is absolutely stable if one of the following two conditions is satisfied:

(I) $\gamma_{i j}=0(i, j=1,2, \ldots, m)$ and $D+U U^{T}<0$.

(II) $\mu_{i}=0(i=1,2, \ldots, m)$ and $D+R R^{T}<0$.

Corollary 4 Under A1, A5, A6, and A7, system (2) is absolutely stable if $\gamma_{i j}=\mu_{i}=0(i, j=$ $1,2, \ldots, m)$ and $D<0$.

The proofs of these corollaries are relatively simple and are omitted here.

Remark 1 It should be pointed out that Lurie system (2) under consideration is an extension of Lurie indirect control systems discussed in $[4,17]$ since the coefficient matrices are norm-unbounded. This is the main feature of this paper. All the theorems and corollaries are applicable to the large-scale time-delay Lurie systems with unbounded coefficients. Particularly, for this class of systems with bounded or constant coefficients, these results are also effective.

\section{Absolute stability of large-scale Lurie systems with multiple nonlinearities}

Consider the following large-scale time-delay Lurie indirect control system with unbounded coefficients and multiple nonlinearities:

$$
\left\{\begin{array}{l}
\dot{x}_{i}(t)=\sum_{j=1}^{m} A_{i j}(t) x_{j}(t)+\sum_{j=1}^{m} B_{i j}(t) x_{j}\left(t-\tau_{j}(t)\right)+\sum_{j=1}^{r} b_{i j}(t) f_{j}\left(\sigma_{j}(t)\right), \\
\quad i=1,2, \ldots, m, \\
\dot{\sigma}_{l}(t)=\sum_{j=1}^{m} c_{l j}^{T}(t) x_{j}(t)-\rho_{l}(t) f_{l}\left(\sigma_{l}(t)\right), \quad l=1,2, \ldots, r
\end{array}\right.
$$

where $x_{i}(t) \in R^{n_{i}}(i=1,2, \ldots, m)$ and $\sigma_{l}(t) \in R(l=1,2, \ldots, r)$ are the state vectors; the vector functions $b_{i j}(t) \in R^{n_{i}}(i=1,2, \ldots, m ; j=1,2, \ldots, r)$ and $c_{l j}(t) \in R^{n_{j}}(l=1,2, \ldots, r ; j=$ 
$1,2, \ldots, m)$ are continuous in $[0, \infty), \sum_{i=1}^{m} n_{i}=n$; the matrix functions $A_{i j}(t), B_{i j}(t) \in R^{n_{i} \times n_{j}}$ $(i, j=1,2, \ldots, m)$ are continuous in $[0, \infty) ; \tau_{j}(t)(j=1,2, \ldots, m)$ refer to the time-delays; $\rho_{l}(t)$ $(l=1,2, \ldots, r)$ is continuous in $[0, \infty)$ and satisfies $\rho_{l}(t) \geq \rho_{l}>0$ with constants and $\rho_{l}$. The continuous nonlinearities $f_{l}\left(\sigma_{l}\right)(l=1,2, \ldots, r)$ satisfy the following sector condition:

$$
F_{\left[k_{l 1}, k_{l 2}\right]}=\left\{f_{l}(\cdot) \mid f_{l}(0)=0 ; k_{l 1} \sigma_{l}^{2}(t) \leq \sigma_{l}(t) f_{l}\left(\sigma_{l}(t)\right) \leq k_{l 2} \sigma_{l}^{2}(t), \sigma_{l}(t) \in R-\{0\}\right\}
$$

where $k_{l 1}, k_{l 2}$ are constants such that $k_{l 2}>k_{l 1}>0$.

Definition 2 System (3) is said to be absolutely stable if its zero solution is globally asymptotically stable for any nonlinearity $f_{i}(\cdot) \in F_{\left[k_{i 1}, k_{i 2}\right]}(i=1,2, \ldots, r)$.

The aforementioned assumptions A1-A3 and the following assumptions are critical to system (3).

A8 For any $t \in[0, \infty)$,

$$
\frac{\left\|P_{i} b_{i j}(t)+\frac{1}{2} c_{j i}(t)\right\|}{\sqrt{\delta_{i}(t) \rho_{j}(t)}} \leq \mu_{i j}
$$

where $\mu_{i j}(i=1,2, \ldots, m ; j=1,2, \ldots, r)$ are constants.

The definitions of matrices $D$ and $R$ are the same as before, whereas the matrix $U$ is redefined as

$$
U=\left[\begin{array}{cccc}
\mu_{11} & \mu_{12} & \cdots & \mu_{1 r} \\
\mu_{21} & \mu_{22} & \cdots & \mu_{2 r} \\
\vdots & \vdots & & \vdots \\
\mu_{m 1} & \mu_{m 2} & \cdots & \mu_{m r}
\end{array}\right]
$$

Theorem 2 Under A1, A2, A3, and A8, system (3) is absolutely stable if $D+R R^{T}+U U^{T}<0$.

Proof Let us construct the following Lyapunov-Krasovskii functional:

$$
V(t, \phi)=\sum_{i=1}^{m}\left(x_{i}^{T}(t) P_{i} x_{i}(t)+\int_{t-\tau_{i}(t)}^{t} x_{i}^{T}(s) G_{i} x_{i}(s) d s\right)+\sum_{i=1}^{r} \int_{0}^{\sigma_{i}(t)} f_{i}(s) d s .
$$

As in the proof of Theorem 1 , we can show that $V(t, \phi)$ satisfies condition (i) of Lemma 2.

Then, taking the time derivative of $V(t, \phi)$ along the trajectory of system (3) yields

$$
\begin{aligned}
& \left.\dot{V}(t, \phi)\right|_{(3)} \\
& =\sum_{i=1}^{m} x_{i}^{T}(t)\left(P_{i} A_{i i}(t)+A_{i i}^{T}(t) P_{i}+G_{i}\right) x_{i}(t)+\sum_{i=1}^{m} \sum_{\substack{j=1 \\
j \neq i}}^{m} x_{i}^{T}(t)\left(P_{i} A_{i j}(t)+A_{j i}^{T}(t) P_{j}\right) x_{j}(t) \\
& \quad+2 \sum_{i=1}^{m} \sum_{j=1}^{m} x_{i}^{T} P_{i} B_{i j}(t) x_{j}\left(t-\tau_{j}(t)\right)+2 \sum_{i=1}^{m} \sum_{j=1}^{r} x_{i}^{T}(t)\left(P_{i} b_{i j}(t)+\frac{1}{2} c_{j i}(t)\right) f_{j}\left(\sigma_{j}(t)\right) \\
& \quad-\sum_{i=1}^{m}\left(1-\dot{\tau}_{i}(t)\right) x_{i}^{T}\left(t-\tau_{i}(t)\right) G_{i} x_{i}\left(t-\tau_{i}(t)\right)-\sum_{i=1}^{r} \rho_{i}(t)\left(f_{i}\left(\sigma_{i}(t)\right)\right)^{2} .
\end{aligned}
$$


Analogously, applying A1, A2, and properties of norms, we obtain

$$
\begin{aligned}
& \left.\dot{V}(t, \phi)\right|_{(3)} \\
& \leq-\sum_{i=1}^{m} \delta_{i}(t)\left\|x_{i}(t)\right\|^{2}+\sum_{i=1}^{m} \sum_{\substack{j=1 \\
j \neq i}}^{m}\left\|P_{i} A_{i j}(t)+A_{j i}^{T}(t) P_{j}\right\|\left\|x_{i}(t)\right\|\left\|x_{j}(t)\right\| \\
& \quad+2 \sum_{i=1}^{m} \sum_{j=1}^{m}\left\|P_{i} B_{i j}(t)\right\|\left\|x_{i}(t)\right\|\left\|x_{j}\left(t-\tau_{j}(t)\right)\right\| \\
& \quad+2 \sum_{i=1}^{m} \sum_{j=1}^{r}\left\|P_{i} b_{i j}(t)+\frac{1}{2} c_{j i}(t)\right\|\left\|x_{i}(t)\right\|\left|f_{j}\left(\sigma_{j}(t)\right)\right| \\
& \quad-\sum_{i=1}^{m}\left(1-\alpha_{i}\right) \lambda_{\min }\left(G_{i}\right)\left\|x_{i}\left(t-\tau_{i}(t)\right)\right\|^{2}-\sum_{i=1}^{r} \rho_{i}(t)\left(f_{i}\left(\sigma_{i}(t)\right)\right)^{2} .
\end{aligned}
$$

By virtue of $\sqrt{\delta_{i}(t)}\left\|x_{i}(t)\right\|, \quad \sqrt{\left(1-\alpha_{i}\right) \lambda_{\min }\left(G_{i}\right)}\left\|x\left(t-\tau_{i}(t)\right)\right\| \quad(i=1,2, \ldots, m)$, and $\sqrt{\rho_{i}(t)}\left|f_{i}\left(\sigma_{i}(t)\right)\right|(i=1,2, \ldots, r)$, we can continue estimating the upper bound of $\left.\dot{V}(t, \phi)\right|_{(3)}$ based on A3 and A8:

$$
\begin{aligned}
\left.\dot{V}(t, \phi)\right|_{(3)} & \\
\leq & -\sum_{i=1}^{m} \delta_{i}(t)\left\|x_{i}(t)\right\|^{2}+\sum_{i=1}^{m} \sum_{\substack{j=1 \\
j \neq i}}^{m} \eta_{i j} \sqrt{\delta_{i}(t)}\left\|x_{i}(t)\right\| \cdot \sqrt{\delta_{j}(t)}\left\|x_{j}(t)\right\| \\
& +\sum_{i=1}^{m} \sum_{j=1}^{m} 2 \gamma_{i j} \sqrt{\delta_{i}(t)}\left\|x_{i}(t)\right\| \cdot \sqrt{\left(1-\alpha_{j}\right) \lambda_{\min }\left(G_{j}\right)}\left\|x_{j}\left(t-\tau_{j}(t)\right)\right\| \\
& +2 \sum_{i=1}^{m} \sum_{j=1}^{r} \mu_{i j}\left\|x_{i}(t)\right\|\left|f_{j}\left(\sigma_{j}(t)\right)\right|-\sum_{i=1}^{m}\left(1-\alpha_{i}\right) \lambda_{\min }\left(G_{i}\right)\left\|x_{i}\left(t-\tau_{i}(t)\right)\right\|^{2} \\
& -\sum_{i=1}^{r} \rho_{i}(t)\left(f_{i}\left(\sigma_{i}(t)\right)\right)^{2} \\
= & Y^{T} \hat{D} Y
\end{aligned}
$$

where

$$
Y=\left[\begin{array}{c}
\sqrt{\delta_{1}(t)}\left\|x_{1}(t)\right\| \\
\vdots \\
\sqrt{\delta_{m}(t)}\left\|x_{m}(t)\right\| \\
\sqrt{\left(1-\alpha_{1}\right) \lambda_{\min }\left(G_{1}\right)}\left\|x_{1}\left(t-\tau_{1}(t)\right)\right\| \\
\vdots \\
\sqrt{\left(1-\alpha_{m}\right) \lambda_{\min }\left(G_{m}\right)}\left\|x_{m}\left(t-\tau_{m}(t)\right)\right\| \\
\sqrt{\rho_{1}(t)}\left|f_{1}\left(\sigma_{1}\right)\right| \\
\vdots \\
\sqrt{\rho_{r}(t)}\left|f_{r}\left(\sigma_{r}\right)\right|
\end{array}\right],
$$




$$
\hat{D}=\left[\begin{array}{cccccccccccc}
-1 & \eta_{12} & \cdots & \eta_{1 m} & \gamma_{11} & \gamma_{12} & \cdots & \gamma_{1 m} & \mu_{11} & \mu_{12} & \cdots & \mu_{1 r} \\
\eta_{21} & -1 & \cdots & \eta_{2 m} & \gamma_{21} & \gamma_{22} & \cdots & \gamma_{2 m} & \mu_{21} & \mu_{22} & \cdots & \mu_{2 r} \\
\vdots & \vdots & & \vdots & \vdots & \vdots & & \vdots & \vdots & \vdots & & \vdots \\
\eta_{m 1} & \eta_{m 2} & \cdots & -1 & \gamma_{m 1} & \gamma_{m 2} & \cdots & \gamma_{m m} & \mu_{m 1} & \mu_{m 2} & \cdots & \mu_{m r} \\
\gamma_{11} & \gamma_{21} & \cdots & \gamma_{m 1} & -1 & 0 & \cdots & 0 & 0 & 0 & \cdots & 0 \\
\gamma_{12} & \gamma_{22} & \cdots & \gamma_{m 2} & 0 & -1 & \cdots & 0 & 0 & 0 & \cdots & 0 \\
\vdots & \vdots & & \vdots & \vdots & \vdots & & \vdots & \vdots & \vdots & & \vdots \\
\gamma_{1 m} & \gamma_{2 m} & \cdots & \gamma_{m m} & 0 & 0 & \cdots & -1 & 0 & 0 & \cdots & 0 \\
\mu_{11} & \mu_{21} & \cdots & \mu_{m 1} & 0 & 0 & \cdots & 0 & -1 & 0 & \cdots & 0 \\
\mu_{12} & \mu_{22} & \cdots & \mu_{m 2} & 0 & 0 & \cdots & 0 & 0 & -1 & \cdots & 0 \\
\vdots & \vdots & & \vdots & \vdots & \vdots & & \vdots & \vdots & \vdots & & \vdots \\
\mu_{1 r} & \mu_{2 r} & \cdots & \mu_{m r} & 0 & 0 & \cdots & 0 & 0 & 0 & \cdots & -1
\end{array}\right] .
$$

Using the preceding notations, we have

$$
\hat{D}=\left[\begin{array}{ccc}
D & R & U \\
R^{T} & -I & 0 \\
U^{T} & 0 & -I
\end{array}\right] .
$$

The following proof is similar to that of Theorem 1. System (3) is absolutely stable if $\hat{D}<0$. Accordingly, the problem comes down to seeking the conditions for $\hat{D}<0$. By Lemma $1, \hat{D}<0$ is equivalent to $D+R R^{T}+U U^{T}<0$. This completes the proof.

Corollary 5 Under A1, A3, A5, and A8, system (3) is absolutely stable if $D+R R^{T}+$ $U U^{T}<0$.

Similarly to the single nonlinearity case, to conclude the absolute stability for system (3), we only need to require that there exists a constant $T \geq 0$ such that the inequality conditions in A2, A3, A5, and A8 are satisfied for $t>T$. Hence, $\mu_{i j}(i=1,2, \ldots, m ; j=1,2, \ldots, r)$ in A 8 can be computed by the corresponding upper limit (if the upper limit is a finite value).

A9

$$
\varlimsup_{t \rightarrow \infty} \frac{\left\|P_{i} b_{i j}(t)+\frac{1}{2} c_{j i}(t)\right\|}{\sqrt{\delta_{i}(t) \rho_{j}(t)}}=\mu_{i j},
$$

where $\mu_{i j}(i=1,2, \ldots, m ; j=1,2, \ldots, r)$ are constants.

Corollary 6 Under A1, A5, A6, and A9, system (3) is absolutely stable if $D+R R^{T}+$ $U U^{T}<0$.

Corollary 7 Under A1, A2, A6, and A9, system (3) is absolutely stable if one of the following two conditions is satisfied:

(I) $\gamma_{i j}=0(i, j=1,2, \ldots, m)$ and $D+U U^{T}<0$.

(II) $\mu_{i j}=0(i=1,2, \ldots, m ; j=1,2, \ldots, r)$ and $D+R R^{T}<0$.

Corollary 8 Under A1, A5, A6, and A9, system (3) is absolutely stable if $\gamma_{i j}=0(i, j=$ $1,2, \ldots, m), \mu_{i j}=0(i=1,2, \ldots, m ; j=1,2, \ldots, r)$, and $D<0$. 
The proofs of these corollaries are relatively simple and are omitted here.

Remark 2 Recently, the absolute stability of time-delay Lurie indirect control systems was studied in $[5,25]$. However, these studies were only applicable to the independent Lurie time-delay systems. Although the authors in [18] considered large-scale Lurie indirect control systems, they did not take the time-delay into account. Therefore, the results in this paper have a greater range of applications.

\section{Numerical simulation}

The following numerical examples are presented to illustrate the effectiveness of the proposed theoretical results.

Example 1 Consider the following large-scale time-delay Lurie indirect control system with a single nonlinearity:

$$
\left\{\begin{aligned}
{\left[\begin{array}{c}
\dot{x}_{2}(t) \\
\dot{x}_{2}(t)
\end{array}\right]=} & {\left[\begin{array}{cc}
-2 t-\frac{1}{2} & 1 \\
t & -3 t-\frac{1}{2}
\end{array}\right]\left[\begin{array}{c}
x_{1}(t) \\
x_{2}(t)
\end{array}\right]+\left[\begin{array}{c}
\sqrt{t} \\
10
\end{array}\right] x_{3}(t) } \\
& +\left[\begin{array}{cc}
\sqrt{\frac{t}{2}} & 0 \\
0 & \sqrt{\frac{t}{6}}
\end{array}\right]\left[\begin{array}{c}
x_{1}\left(t-\tau_{1}(t)\right) \\
x_{2}\left(t-\tau_{1}(t)\right)
\end{array}\right]+\left[\begin{array}{c}
t^{\frac{1}{3}} \\
0
\end{array}\right] x_{3}\left(t-\tau_{2}(t)\right) \\
& +\left[\begin{array}{c}
-\frac{t}{2} \\
0
\end{array}\right] f(\sigma(t)), \\
\dot{x}_{3}(t)= & {[\sqrt{t} \sqrt{t}]\left[\begin{array}{c}
x_{1}(t) \\
x_{2}(t)
\end{array}\right]-\left(t+\frac{1}{2}\right) x_{3}(t)+\left[\begin{array}{l}
\frac{1}{4} \\
4
\end{array}\right]\left[\begin{array}{l}
x_{1}\left(t-\tau_{1}(t)\right) \\
x_{2}\left(t-\tau_{1}(t)\right)
\end{array}\right] } \\
& +\sqrt{\frac{t}{8}} x_{3}\left(t-\tau_{2}(t)\right)-t f(\sigma(t)), \\
\dot{\sigma}(t)= & {[t \sqrt{t}]\left[\begin{array}{c}
x_{1}(t) \\
x_{2}(t)
\end{array}\right]+2 t x_{3}(t)-(t+1) f(\sigma(t)), }
\end{aligned}\right.
$$

where $\tau_{1}(t)=3+0.5 \sin t, \tau_{2}(t)=7$, and $f(\cdot) \in F_{[0.01,100]}$.

This system is of the form (2) with

$$
\begin{aligned}
& A_{11}(t)=\left[\begin{array}{cc}
-2 t-\frac{1}{2} & 1 \\
t & -3 t-\frac{1}{2}
\end{array}\right], \quad A_{12}(t)=\left[\begin{array}{c}
\sqrt{t} \\
10
\end{array}\right], \\
& A_{21}(t)=\left[\begin{array}{ll}
\sqrt{t} & \sqrt{t}
\end{array}\right], \quad A_{22}(t)=-t-\frac{1}{2}, \\
& B_{11}(t)=\left[\begin{array}{cc}
\sqrt{\frac{t}{2}} & 0 \\
0 & \sqrt{\frac{t}{6}}
\end{array}\right], \quad B_{12}(t)=\left[\begin{array}{c}
t^{\frac{1}{3}} \\
0
\end{array}\right], \quad B_{21}(t)=\left[\begin{array}{ll}
0 & t^{\frac{1}{4}}
\end{array}\right], \quad B_{22}(t)=\sqrt{\frac{t}{8}}, \\
& b_{1}(t)=\left[\begin{array}{c}
-\frac{t}{2} \\
0
\end{array}\right], \quad b_{2}(t)=-t, \quad c_{1}(t)=\left[\begin{array}{c}
t \\
\sqrt{t}
\end{array}\right], \quad c_{2}(t)=2 t, \quad \rho(t)=t+1 .
\end{aligned}
$$

This problem cannot be solved by the method of [18], which did not deal with the timedelay case. Now, we use the criteria proposed in this paper to analyze the absolute stability of this system.

Clearly, A1 is satisfied with $\tau_{1}=3.5, \alpha_{1}=0.5, \tau_{2}=7, \alpha_{2}=0$.

Then, by letting $P_{1}=G_{1}=I$ we have

$$
P_{1} A_{11}(t)+A_{11}^{T}(t) P_{1}+G_{1}=\left[\begin{array}{cc}
-4 t & t+1 \\
t+1 & -6 t
\end{array}\right]
$$


and

$$
\lambda\left(P_{1} A_{11}(t)+A_{11}^{T}(t) P_{1}+G_{1}\right) \leq-5 t+\sqrt{2 t^{2}+2 t+1} .
$$

Taking $T=\sqrt{2}$, for $t>T$, we obtain

$$
\lambda\left(P_{1} A_{11}(t)+A_{11}^{T}(t) P_{1}+G_{1}\right)<-5 t+\sqrt{2}(t+1)<-(4-\sqrt{2}) t .
$$

Thus, we can take

$$
\delta_{1}(t)=(4-\sqrt{2}) t
$$

Notice that, for $t>T$, we have the inequality

$$
-\delta_{1}(t) \leq-\xi_{1}=-(4 \sqrt{2}-2) .
$$

Letting $P_{2}=G_{2}=1$, we obtain

$$
P_{2} A_{22}(t)+A_{22}^{T}(t) P_{2}+G_{2}=-2 t .
$$

Then, we can take

$$
\delta_{2}(t)=2 t .
$$

Similarly, for $t>T$, we have the inequality

$$
-\delta_{2}(t) \leq-\xi_{2}=-2 \sqrt{2}
$$

Hence A2 is satisfied. In addition, we get

$$
\begin{aligned}
& \lim _{t \rightarrow \infty} \frac{\left\|P_{1} A_{12}(t)+A_{21}^{T}(t) P_{2}\right\|}{\sqrt{\delta_{1}(t) \delta_{2}(t)}}=0, \\
& \lim _{t \rightarrow \infty} \frac{\left\|P_{2} A_{21}(t)+A_{12}^{T}(t) P_{1}\right\|}{\sqrt{\delta_{2}(t) \delta_{1}(t)}}=0 .
\end{aligned}
$$

Moreover, we have

$$
\begin{aligned}
& \lim _{t \rightarrow \infty} \frac{\left\|P_{1} B_{11}(t)\right\|}{\sqrt{\delta_{1}(t)\left(1-\alpha_{1}\right) \lambda_{\min }\left(G_{1}\right)}}=\frac{1}{\sqrt{4-\sqrt{2}}}, \\
& \lim _{t \rightarrow \infty} \frac{\left\|P_{1} B_{12}(t)\right\|}{\sqrt{\delta_{1}(t)\left(1-\alpha_{2}\right) \lambda_{\min }\left(G_{2}\right)}}=0, \\
& \lim _{t \rightarrow \infty} \frac{\left\|P_{2} B_{21}(t)\right\|}{\sqrt{\delta_{2}(t)\left(1-\alpha_{1}\right) \lambda_{\min }\left(G_{1}\right)}}=0, \\
& \lim _{t \rightarrow \infty} \frac{\left\|P_{2} B_{22}(t)\right\|}{\sqrt{\delta_{2}(t)\left(1-\alpha_{2}\right) \lambda_{\min }\left(G_{2}\right)}}=\frac{1}{4} .
\end{aligned}
$$

Thus, A6 is satisfied with $\eta_{12}=0, \eta_{21}=0, \gamma_{11}=\frac{1}{\sqrt{4-\sqrt{2}}}, \gamma_{12}=0, \gamma_{21}=0, \gamma_{22}=\frac{1}{4}$. 
Figure 1 The state response of system (4).

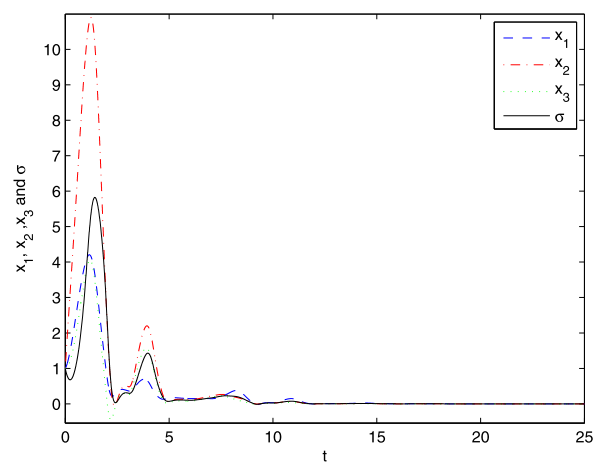

Furthermore, we derive

$$
\begin{aligned}
& \lim _{t \rightarrow \infty} \frac{\left\|P_{1} b_{1}(t)+\frac{1}{2} c_{1}(t)\right\|}{\sqrt{\delta_{1}(t) \rho(t)}}=0, \\
& \lim _{t \rightarrow \infty} \frac{\left\|P_{2} b_{2}(t)+\frac{1}{2} c_{2}(t)\right\|}{\sqrt{\delta_{2}(t) \rho(t)}}=0 .
\end{aligned}
$$

Hence A7 is satisfied with $\mu_{1}=\mu_{2}=0$.

Finally, we verify that

$$
D+R R^{T}=\left[\begin{array}{cc}
\frac{\sqrt{2}-10}{14} & 0 \\
0 & -\frac{15}{16}
\end{array}\right]<0 .
$$

Thus, we conclude from Corollary 3 that system (4) is absolutely stable.

For simulation, we choose $f(\sigma(t))=2 \sigma(t)+\sin \sigma(t)$ and $\left[x_{1}(s) x_{2}(s) x_{3}(s) \sigma(0)\right]^{T}=$ $\left[\begin{array}{llll}1 & 1 & 1 & 1\end{array}\right]^{T}, s \in[-7,0]$. The numerical simulation result is shown in Figure 1.

It is seen in Figure 1 that the states of system (4) converge to zero asymptotically. Thus the effectiveness of the proposed criteria is illustrated by the simulation result.

Example 2 Consider the following large-scale time-delay Lurie indirect control system with two nonlinearities:

$$
\left\{\begin{aligned}
{\left[\begin{array}{c}
\dot{x}_{1}(t) \\
\dot{x}_{2}(t)
\end{array}\right]=} & {\left[\begin{array}{cc}
-4 t-\frac{1}{2} & 1 \\
0 & -3 t-\frac{1}{2}
\end{array}\right]\left[\begin{array}{c}
x_{1}(t) \\
x_{2}(t)
\end{array}\right]+\left[\begin{array}{c}
\sqrt{t} \\
0
\end{array}\right] x_{3}(t) } \\
& +\left[\begin{array}{cc}
\sqrt{t} & 0 \\
0 & \sqrt{\frac{t}{6}}
\end{array}\right]\left[\begin{array}{l}
x_{1}\left(t-\tau_{1}(t)\right) \\
x_{2}\left(t-\tau_{1}(t)\right)
\end{array}\right]+\left[\begin{array}{c}
t^{\frac{1}{4}} \\
10
\end{array}\right] x_{3}\left(t-\tau_{2}(t)\right) \\
& +\left[\begin{array}{c}
-t \\
0
\end{array}\right] f_{1}\left(\sigma_{1}(t)\right)+\left[\begin{array}{c}
-t \\
0
\end{array}\right] f_{2}\left(\sigma_{2}(t)\right), \\
\dot{x}_{3}(t)= & {\left[\begin{array}{l}
1 \sqrt{t}
\end{array}\right]\left[\begin{array}{c}
x_{1}(t) \\
x_{2}(t)
\end{array}\right]-\left(t+\frac{1}{2}\right) x_{3}(t)+\left[\begin{array}{l}
1 \\
0
\end{array}\right]\left[\begin{array}{l}
x_{1}\left(t-\tau_{1}(t)\right) \\
x_{2}\left(t-\tau_{1}(t)\right)
\end{array}\right] } \\
& +\frac{\sqrt{t}}{4} x_{3}\left(t-\tau_{2}(t)\right)-t f_{1}\left(\sigma_{1}(t)\right)+2 t f_{2}\left(\sigma_{2}(t)\right), \\
\dot{\sigma}_{1}(t)= & {[2 t 2 \sqrt{t}]\left[\begin{array}{l}
x_{1}(t) \\
x_{2}(t)
\end{array}\right]+4 t x_{3}(t)-(t+1) f_{1}\left(\sigma_{1}(t)\right), } \\
\dot{\sigma}_{2}(t)= & {[2 t 0]\left[\begin{array}{l}
x_{1}(t) \\
x_{2}(t)
\end{array}\right]-6 t x_{3}(t)-(3 t+1) f_{2}\left(\sigma_{2}(t)\right), }
\end{aligned}\right.
$$

where $\tau_{1}(t)=3+0.5 \sin t, \tau_{2}(t)=7$, and $f(\cdot) \in F_{[0.01,100]}$. 
In comparison with system (3), the coefficient matrices here are

$$
\begin{aligned}
& A_{11}(t)=\left[\begin{array}{cc}
-4 t-\frac{1}{2} & 1 \\
0 & -3 t-\frac{1}{2}
\end{array}\right], \quad A_{12}(t)=\left[\begin{array}{c}
\sqrt{t} \\
0
\end{array}\right], \\
& A_{21}(t)=\left[\begin{array}{ll}
1 & \sqrt{t}
\end{array}\right], \quad A_{22}(t)=-t-\frac{1}{2}, \\
& B_{11}(t)=\left[\begin{array}{cc}
\sqrt{t} & 0 \\
0 & \sqrt{\frac{t}{6}}
\end{array}\right], \quad B_{12}(t)=\left[\begin{array}{c}
t^{\frac{1}{4}} \\
10
\end{array}\right], \quad B_{21}(t)=\left[\begin{array}{ll}
0 & t^{\frac{1}{4}}
\end{array}\right], \quad B_{22}(t)=\frac{\sqrt{t}}{4}, \\
& b_{11}(t)=\left[\begin{array}{c}
-t \\
0
\end{array}\right], \quad b_{12}(t)=\left[\begin{array}{c}
-t \\
0
\end{array}\right], \quad b_{21}(t)=-t, \quad b_{22}(t)=2 t, \\
& c_{11}(t)=\left[\begin{array}{c}
2 t \\
2 \sqrt{t}
\end{array}\right], \quad c_{12}(t)=4 t, \quad c_{21}(t)=\left[\begin{array}{c}
2 t \\
0
\end{array}\right], \quad c_{22}(t)=-6 t, \\
& \rho_{1}(t)=t+1, \quad \rho_{2}(t)=3 t+1 .
\end{aligned}
$$

Next, we show that this system satisfies the conditions of Corollary 6 .

Clearly, A1 is satisfied with $\tau_{1}=3.5, \alpha_{1}=0.5, \tau_{2}=7, \alpha_{2}=0$. Also, letting $P_{1}=G_{1}=I$, we obtain

$$
P_{1} A_{11}(t)+A_{11}^{T}(t) P_{1}+G_{1}=\left[\begin{array}{cc}
-8 t & 1 \\
1 & -6 t
\end{array}\right]
$$

and

$$
\lambda\left(P_{1} A_{11}(t)+A_{11}^{T}(t) P_{1}+G_{1}\right) \leq-7 t+\sqrt{t^{2}+1} .
$$

Taking $T=1$, for $t>T$, we have

$$
\lambda\left(P_{1} A_{11}(t)+A_{11}^{T}(t) P_{1}+G_{1}\right)<-(7-\sqrt{2}) t .
$$

Similarly, letting $P_{2}=G_{2}=1$, we have

$$
P_{2} A_{22}(t)+A_{22}^{T}(t) P_{2}+G_{2}=-2 t .
$$

Thus A5 is fulfilled with $\delta_{1}(t)=(7-\sqrt{2}) t, \delta_{2}(t)=2 t$. Moreover,

$$
\begin{aligned}
& \lim _{t \rightarrow \infty} \frac{\left\|P_{1} A_{12}(t)+A_{21}^{T}(t) P_{2}\right\|}{\sqrt{\delta_{1}(t) \delta_{2}(t)}}=0, \\
& \lim _{t \rightarrow \infty} \frac{\left\|P_{2} A_{21}(t)+A_{12}^{T}(t) P_{1}\right\|}{\sqrt{\delta_{2}(t) \delta_{1}(t)}}=0 .
\end{aligned}
$$


In addition, by simple calculation we get

$$
\begin{aligned}
& \lim _{t \rightarrow \infty} \frac{\left\|P_{1} B_{11}(t)\right\|}{\sqrt{\delta_{1}(t)\left(1-\alpha_{1}\right) \lambda_{\min }\left(G_{1}\right)}}=\sqrt{\frac{2}{7-\sqrt{2}}}, \\
& \lim _{t \rightarrow \infty} \frac{\left\|P_{1} B_{12}(t)\right\|}{\sqrt{\delta_{1}(t)\left(1-\alpha_{2}\right) \lambda_{\min }\left(G_{2}\right)}}=0, \\
& \lim _{t \rightarrow \infty} \frac{\left\|P_{2} B_{21}(t)\right\|}{\sqrt{\delta_{2}(t)\left(1-\alpha_{1}\right) \lambda_{\min }\left(G_{1}\right)}}=0, \\
& \lim _{t \rightarrow \infty} \frac{\left\|P_{2} B_{22}(t)\right\|}{\sqrt{\delta_{2}(t)\left(1-\alpha_{2}\right) \lambda_{\min }\left(G_{2}\right)}}=\frac{1}{4 \sqrt{2}} .
\end{aligned}
$$

Hence A6 is satisfied with $\eta_{12}=\eta_{21}=0, \gamma_{11}=\sqrt{\frac{2}{7-\sqrt{2}}}, \gamma_{12}=0, \gamma_{21}=0, \gamma_{22}=\frac{1}{4 \sqrt{2}}$.

Furthermore, we have

$$
\begin{aligned}
& \lim _{t \rightarrow \infty} \frac{\left\|P_{1} b_{11}(t)+\frac{1}{2} c_{11}(t)\right\|}{\sqrt{\delta_{1}(t) \rho_{1}(t)}}=0, \\
& \lim _{t \rightarrow \infty} \frac{\left\|P_{1} b_{12}(t)+\frac{1}{2} c_{21}(t)\right\|}{\sqrt{\delta_{1}(t) \rho_{2}(t)}}=0, \\
& \lim _{t \rightarrow \infty} \frac{\left\|P_{2} b_{21}(t)+\frac{1}{2} c_{12}(t)\right\|}{\sqrt{\delta_{2}(t) \rho_{1}(t)}}=\frac{1}{\sqrt{2}}, \\
& \lim _{t \rightarrow \infty} \frac{\left\|P_{2} b_{22}(t)+\frac{1}{2} c_{22}(t)\right\|}{\sqrt{\delta_{2}(t) \rho_{2}(t)}}=\frac{1}{\sqrt{6}} .
\end{aligned}
$$

Then A9 is satisfied with $\mu_{11}=\mu_{12}=0, \mu_{21}=\frac{1}{\sqrt{2}}, \mu_{22}=\frac{1}{\sqrt{6}}$. At last, we verify that

$$
D+R R^{T}+U U^{T}=\left[\begin{array}{cc}
\frac{\sqrt{2}-5}{7-\sqrt{2}} & 0 \\
0 & -\frac{29}{96}
\end{array}\right]<0 .
$$

Thus, according to Corollary 6 , system (5) is absolutely stable.

For simulation, we choose

$$
f_{1}(\sigma(t))=2 \sigma(t)+\sin \sigma(t), \quad f_{2}(\sigma(t))= \begin{cases}\sigma(t), & |\sigma(t)|<1, \\ \sigma^{3}(t), & 1 \leq|\sigma(t)| \leq 2 \\ 4 \sigma(t), & |\sigma(t)|>2,\end{cases}
$$

and $\left[x_{1}(s) x_{2}(s) x_{3}(s) \sigma_{1}(0) \sigma_{2}(0)\right]^{T}=\left[\begin{array}{lllll}1 & 1 & 1 & 1 & 1\end{array}\right]^{T}, s \in[-7,0]$. The response curves of this system are shown in Figure 2.

As depicted in Figure 2, system (5) is asymptotically stable even though the system coefficients are unbounded.

\section{Conclusion}

In this paper, we have investigated the absolute stability problem of time-varying largescale time-delay Lurie indirect control systems. Based on the second Lyapunov method, 
Figure 2 The state response of system (5).

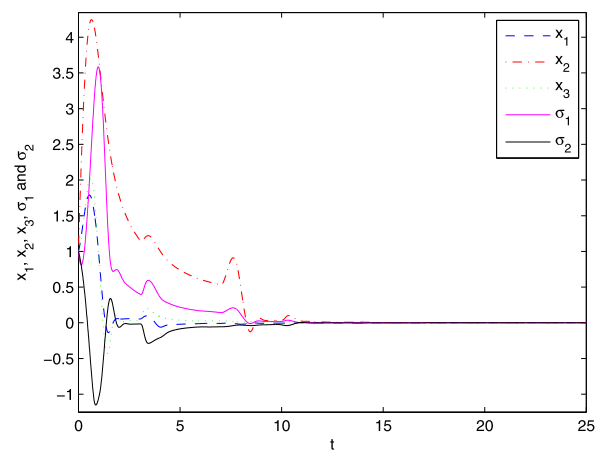

to guarantee absolute stability of this class of systems, some sufficient conditions were formulated by simple inequalities. Finally, two numerical examples were presented to show the effectiveness of the proposed methods.

\section{Acknowledgements}

This work was supported by the Oriented Award Foundation for Science and Technological Innovation, Inner Mongolia Autonomous Region, China.

\section{Competing interests}

The authors declare that they have no competing interests.

\section{Authors' contributions}

The authors have made the same contribution. Both authors read and approved the final manuscript.

\section{Publisher's Note}

Springer Nature remains neutral with regard to jurisdictional claims in published maps and institutional affiliations.

Received: 18 February 2017 Accepted: 31 August 2017 Published online: 11 September 2017

\section{References}

1. Lurie, Al, Postnikov, VN: On the theory of stability of control system. Prikl. Mat. Meh. 8(3), 283-286 (1944)

2. Aizerman, MA, Gantmaher, FR: Absolute Stability of Regulator Systems. Holden-Day, San Francisco (1964)

3. Lurie, Al: Some Nonlinear Problems in the Theory of Automatic Control. H.M. Stationary Office, London (1957)

4. Liao, X, Yu, P: Absolute Stability of Nonlinear Control Systems. Springer, New York (2008)

5. Shatyrko, A, Diblík, J, Khusainov, D, Růžičková, M: Stabilization of Lur'e-type nonlinear control systems by Lyapunov-Krasovskii functionals. Adv. Differ. Equ. 2012, 229 (2012)

6. Liberzon, MR: Essays on the absolute stability theory. Autom. Remote Control 67(10), 1610-1644 (2006)

7. Gao, J, Su, H, Ji, X, Chu, J: New delay-dependent absolute stability criteria for Lurie control systems. Acta Autom. Sin. 34(10), 1275-1280 (2008)

8. Qiu, F, Zhang, Q: Absolute stability analysis of Lurie control system with multiple delays: an integral-equality approach. Nonlinear Anal., Real World Appl. 12(3), 1475-1484 (2011)

9. Zeng, H-B, He, Y, Wu, M, Feng, Z-Y: New absolute stability criteria for Lurie nonlinear systems with time-varying delay. Control Decis. 25(3) 346-350 (2010)

10. Huang, $\mathrm{H}, \mathrm{CaO}, \mathrm{J}$ : Master-slave synchronization of Lur'e systems based on time-varying delay feedback control. Int. J. Bifurc. Chaos 17(11), 4159-4166 (2007)

11. Lu, J, Cao, J, Ho, DWC: Adaptive stabilization and synchronization for chaotic Lur'e systems with time-varying delay. IEEE Trans. Circuits Syst. I, Regul. Pap. 55(5), 1347-1356 (2008)

12. Huang, H, Feng, G, Cao, J: Exponential synchronization of chaotic Lur'e systems with delayed feedback control. Nonlinear Dyn. 57(3), 441-453 (2009)

13. He, W, Qian, F, Han, Q-L, Cao, J: Synchronization error estimation and controller design for delayed Lur'e systems with parameter mismatches. IEEE Trans. Neural Netw. Learn. Syst. 23(10), 1551-1563 (2012)

14. Cao, J, Sivasamy, R, Rakkiyappan, R: Sampled-data $H_{\infty}$ synchronization of chaotic Lur'e systems with time delay. Circuits Syst. Signal Process. 35(3), 811-835 (2016)

15. Siljak, DD: Large-Scale Dynamic Systems: Stability and Structure. North Holland, Amsterdam (1978)

16. Nian, X, Li, X, Yang, Y, Zuo, Z: Bilinear matrix inequality approach to the absolute stability of interconnected Lurie control systems. Control Theory Appl. 22(6), 999-1004 (2005)

17. Guo, J, Liao, F: Absolute stability of Lurie indirect control large-scale systems. J. Univ. Sci. Technol. Beijing 28(7), 704-708 (2006)

18. Liao, F, Li, A, Sun, F: Absolute stability of Lurie systems and Lurie large-scale systems with multiple operators and unbounded coefficients. J. Univ. Sci. Technol. Beijing 31(11), 1472-1479 (2009) 
19. Wang, D, Liao, F: Absolute stability of Lurie direct control systems with time-varying coefficients and multiple nonlinearities. Appl. Math. Comput. 219(9), 4465-4473 (2013)

20. Liao, F, Wang, D: Absolute stability criteria for large-scale Lurie direct control systems with time-varying coefficients. Sci. World J. 2014, Article ID 631604 (2014). doi:10.1155/2014/631604

21. Boyd, S, Ghaoui, LE, Feron, E, Balakrishnan, V: Linear Matrix Inequality in Systems and Control Theory. SIAM, Philadelphia (1994)

22. Burton, TA: Volterra Integral and Differential Equations. Academic Press, New York (1983)

23. Burton, TA: Uniform asymptotical stability in functional differential equations. Proc. Am. Math. Soc. 68(2), 195-199 (1978)

24. Lefschetz, S: Stability of Nonlinear Control Systems. Academic Press, New York (1965)

25. Liao, F, Yu, X, Deng, J: Absolute stability of time-varying delay Lurie indirect control systems with unbounded coefficients. Adv. Differ. Equ. 2017, 38 (2017)

\section{Submit your manuscript to a SpringerOpen ${ }^{\circ}$} journal and benefit from:

- Convenient online submission

- Rigorous peer review

- Open access: articles freely available online

- High visibility within the field

- Retaining the copyright to your article

Submit your next manuscript at $\gg$ springeropen.com 